\title{
At the Crossroads of One Health: Lessons Learned from Foot and Mouth Disease in COVID-19 Pandemic
}

\author{
Bramhadev Pattnaik ${ }^{1}$, Sharanagouda S Patil ${ }^{2}$, Raghavendra G. Amachawadi ${ }^{3}$, Awadh B Pandey ${ }^{4}$, A. P. \\ Dash ${ }^{5}$, Mahendra P Yadav ${ }^{6}$, Ashwini Prasad ${ }^{7}$, Latha K.C ${ }^{8}$, Kollur Shiva Prasad ${ }^{\text {, }}$ \\ Chandan Shivamallu ${ }^{10, *}$
}

1 Former Director, ICAR- Directorate of Foot and Mouth Disease, Mukteswar, India 263138

2 ICAR-National Institute of Veterinary Epidemiology and Disease Informatics (NIVEDI), Bengaluru, Karnataka, India

3 Department of Clinical Sciences, College of Veterinary Medicine, Kansas State University, Manhattan, KS, United States of America

4 ICAR-Indian Veterinary Research Institute, Izatnagar, Bareilly, U.P, India

5 Former Advisor, WHO-SEARO \& Vice-Chancellor, CUTN, Tamil Nadu, India

6 Former Vice-Chancellor, SVP University of Agriculture \& Technology, Meerut, India 250110

7 Department of Microbiology, School of Life Sciences, JSS Academy of Higher Education and Research, Mysuru, Karnataka - 570 015, India

8 University Grant Commission, UGC- South Western Regional Office, Palace Road, Bangalore-560009, Karnataka, India.

9 Department of Sciences, Amrita School of Arts and Sciences, Amrita Vishwa Vidyapeetham, Mysuru Campus, Mysuru, Karnataka - 570 026, India

10 Department of Biotechnology and Bioinformatics, School of Life Sciences, JSS Academy of Higher Education and Research, Mysuru, Karnataka - 570 015, India

* Correspondence: chandans@jssuni.edu.in (C.S);

Received: 18.03.2021; Revised: 25.06.2021; Accepted: 30.06.2021; Published: 5.09.2021

Abstract: Foot-and-mouth disease (FMD) and Coronavirus Disease 2019 (COVID-19) are transboundary diseases caused by single-stranded positive-sense RNA viruses with similarities in genome replication and viral protein synthesis. In FMD, asymptomatic infection leads to carrier status and persistently infected animals that threaten the animals vaccinated with a trivalent inactivated whole virus vaccine. Similar information on COVID-19 is not yet available. As COVID-19 vaccination is introduced in January 2021 (since 16 January 2021 in India), its outcome can be assessed by the yearend; and while doing so, the experiences gained in the control of FMD in livestock world wide can be applied, including monitoring of vaccination response, duration of immunity, level of herd immunity developed, and antigenic matching of the vaccine virus. Antigenic divergence of the virus is a major issue in FMD, and different geographical regions in the world use different virus strains in vaccine preparations to antigenically match circulating virus strains in respective regions for control of the disease. Non-synonymous mutations in the critical antigenic determinants of SARS-CoV-2 have been observed, and there is likely the existence/ development of antigenic variants. Therefore, during the post-COVID-19 vaccination regime, it will be essential to monitor the suitability of the in-use vaccine strain region-wise from time to time, as there could be an eruption of isolated outbreaks in a country arising due to antigenic variation and variants. In the context of the present scenario of COVID-19 around the Globe and multiple ongoing efforts to develop suitable vaccine(s) to control the disease, it is a must to develop NSP-antibody (that differentiate infected from vaccinated) assays to differentiate infected from vaccinated individuals(DIVI; DIVA in veterinary epidemiology). The techniques used and experiences gained in ongoing FMD control programs in the endemic countries can be applied to COVID-19 control in a country; and finally, the Globe. After achieving the control of COVID-19, the aim would be to eradicate the virus, which will be tough even with vaccination, as the disease/ infection may become endemic during the time to come. To achieve this, applying the principles of Progressive 
Control Pathway for Foot-and-Mouth Disease (PCP-FMD; FAO/OIE) to COVID-19 control will be beneficial in its control. The present review discusses the issue of control of COVID-19.

Keywords: COVID-19; FMD; RNA virus; Progressive control pathway-FMD; NSP; Vaccine; DIVA/DIVI; One Health

(C) 2021 by the authors. This article is an open-access article distributed under the terms and conditions of the Creative Commons Attribution (CC BY) license (https://creativecommons.org/licenses/by/4.0/).

\section{Introduction}

FMD (Foot-and-mouth disease) dates back to a description of the disease by a monk, Hieronymous Fracastorius, who in 1546 described an epidemic that occurred in cattle near Verona, Italy [1]. In the late nineteenth century (1897-98), it was shown by Loeffler and Frosch [2] working in a German laboratory (Greisswald and Insel Rheims) that the agent causing the disease was a virus. This is the first virus of vertebrates to be discovered soon after the discovery of the tobacco mosaic virus of plants. In 1989, with a complete description of the three-dimensional structure of the virion [3], the FMD virus was classified as a species in the Aphthovirus genus of the family Picornaviridae. Coronavirus $(\mathrm{CoV})$ is known to exist earlier than Aphthovirus, and infection with bovine coronavirus $(\mathrm{BCoV})$ has been recorded both in cattle and humans [4]. In the 1890s, human coronavirus OC43 diverged from bovine coronavirus after a cross-species spillover event. It is speculated that the flu pandemic of 18891890 may have been caused by the BCoV spillover event and not by the influenza virus [5]. The present Coronavirus Disease 2019 (COVID-19) caused by the SARS-CoV-2 since December 2019 (first case of COVID-19 in India on 30 January 2020) is the third documented spillover of an animal coronavirus to humans (zoonoses) in two decades that has resulted in a major Global pandemic [6]. The substantial degree of genetic heterogeneity of $15 \%$ accumulated among human isolates of SARS-CoV-2 is not surprising for an RNA virus that has been evolving over a short time [7,8]. As a typical RNA virus, the average evolutionary rate of $\mathrm{CoVs}$ is about $10^{-4}$ nucleotide substitutions per site per year, with mutations arising during every replication cycle [9]. There is an urgent need to develop an appropriate monitoring system for undertaking molecular surveillance to elucidate the mutation and transmission capability of SARS-CoV-2 [7]. Both FMD and COVID-19 are transboundary diseases caused by single-stranded positive-sense RNA viruses with similarity in genome replication and viral protein synthesis; the former virus has no envelope, unlike the latter. RNA viruses are prone to replication error(s), leading to the generation of non-identical sequences, and competitive selection leading to the predominance of certain sequences [10]. Both the diseases have a short incubation period. Both viruses efficiently excrete as respiratory droplets/ mist, infect the host(s) primarily through the upper respiratory tract, and cause clinical and sub-clinical/ asymptomatic disease (Table 1). In FMD, asymptomatic infection leads to carrier status in animals that threaten to vaccinate (with a trivalent inactivated whole virus vaccine) animals as FMD vaccination does not protect from virus infection (superinfection). Similar information on COVID-19 is not yet available. Once COVID-19 vaccination reaches year-end, the outcome can be assessed; and while doing so, the experiences gained in the control of FMD in livestock worldwide can be applied, including monitoring of vaccination response, duration of immunity, level of herd immunity developed, antigenic matching of the vaccine virus (with the outbreak/ field strains) from time to time to monitor the efficacy of the vaccine over natural mutations occurring in the circulating virus strains, and differentiation of vaccinated from infected humans. The duration and nature of immunity generated in response to SARS-CoV-2 infection 
are unknown, and it is assumed that primary infection may protect humans from subsequent infection for some time [11]. However, such protection from the subsequent infection will be limited to antigenically similar virus strains and may not be against antigenic variants. Mutations in SARS-CoV-2 at important positions are being reported, which is mentioned later. Antigenic divergence of the virus is a major issue in FMD. Different geographical regions use different virus strains in the inactivated vaccine preparations to antigenically match circulating virus strains in respective regions. Non-synonymous mutations in the critical antigenic determinants of SARS-CoV-2 have been observed, and there is likely the existence of antigenic variants. Therefore, during post-COVID-19 vaccination, it will be essential to monitor the suitability of the in-use vaccine strain regionally, as there could be the eruption of isolated outbreaks in a country arising from antigenic variation in circulating virus strains. The techniques used and experiences gained in FMD control programs can be applied to COVID19 control in a country; finally, the Globe. Following control of COVID-19, the aim would be to eradicate the virus, which will be tough even with vaccination. To achieve this, applying the principles of Progressive Control Pathway for Foot-and-Mouth Disease (PCP-FMD; FAO/OIE) to COVID-19 control will be beneficial, as described later in the text.

\section{SARS-CoV-2 and COVID-19}

The duration and nature of immunity generated in response to SARS-CoV-2 infection are unknown yet [11]. It is assumed that COVID-19 infection results in an immune response that protects individuals from future infections or illness for some amount of time. The duration of such protection is a critical determinant of the outcome of subsequent exposure to the virus. The presence or absence of protective immunity due to infection/ vaccination (when available) will impact virus transmission and disease severity. In the case of FMD, vaccinated animals are susceptible to superinfection as the current polyvalent vaccine does not impart sterile immunity; however, vaccinated animals are protected from clinical sickness and pose a barrier to virus transmission, thereby controlling the disease in a vaccinated herd. Protection from FMD is imparted by herd immunity and not by individual animal immunity. To impart herd immunity, a minimum of $80 \%$ of animals need to be vaccinated regularly at 6 months intervals. Further, immunity can be breached by antigenic variants to cause clinical disease. However, it is essential to look at the protective/ virus-neutralizing antibodies in the herd in case of clinical disease in the vaccinated population. Therefore, the vaccinated animal herds are regularly examined for the level of protective antibodies in serum and antibodies to non-structural protein(s) to monitor the possible circulation of the virus in vaccinated animals. It is always possible that some FMD vaccinated herds in spite of having protective vaccinal immunity, may also have anti-NSP antibodies arising mostly due to superinfection and sometimes due to impure vaccine formulation. The situation could be the same in the case of COVID-19 once control by vaccination is taken up/ implemented. Applying NSP antibody assay(s) will be easier as the current Covid-19 vaccines are either subunit vaccines having Spike gene sequence or inactivated whole virus vaccine. The virus SARS-CoV-2 has about 29829 bases with 12 coding regions (ORFs), viz., 1ab, S, 3, E, M, 7, 8, 9, 10b, N, 13, and 14 [9]. The four structural proteins are $\mathrm{S}$ (spike), $\mathrm{E}$ (envelope), $\mathrm{M}$ (membrane), and $\mathrm{N}$ (nucleoprotein). The remaining are non-structural proteins (NSPs). The polyprotein $1 \mathrm{ab}$ (pp1ab) is the largest protein of coronaviruses that, through proteolytic cleavage, is divided into 16 mature non-structural proteins (NSPs). There are 10 major NSPs coded by the ORF 1ab of SARS-CoV-2. There are 
other NSPs and 9 accessory proteins coded by other ORFs. The NSPs are involved in replicating and transcribing the viral genome and are responsible for the cleavage of the viral polyproteins. The NSPs are antigenic and elicit antibody responses against themselves, and detection of these antibodies (antibodies against NSPs) can differentiate infection/ superinfection from vaccination. There will be no detectable NSP antibodies in humans vaccinated with purified COVID-19 vaccine preparations lacking NSPs. The presence of antibodies to an NSP or more is indicative of virus infection. In the context of the present scenario of COVID-19 around the Globe, and vaccination drive to control the disease, it is necessary to develop NSP-antibody assays to differentiate immunity elicited by vaccination from that of virus infection. So also, NSP-antibody assays hold promise in understanding and estimating the spread of the virus/ infection. The selected NSPs of the SARS-CoV-2 viz., L protein, 3C like protease, RdRp, helicase, and single-strand RNA binding protein, etc. (virus infection associated antigens; VIA antigens) can be used to develop assays for detection of virus -infection associated (VIA) antibodies. This VIA approach is currently being extensively used to monitor the control of FMD; a disease of livestock caused by a positive-sense singlestranded RNA virus known as Apthovirus in the family Picornaviridae through regular vaccination in FMD endemic countries in the world. Clearance of the virus from the animal population is indicated by the absence of antibodies to NSPs. This VIA approach will be helpful in not only monitoring the spread of the virus and also, but monitor clearance of the virus from COVID-19 also vaccinated areas. Antibodies against structural proteins (SP) alone cannot reveal the status/ stage of COVID control or the vaccine's efficacy in eliminating the virus from the population. Therefore, NSP-antibody assays must be in place as companion diagnostics to elucidate the rate of success of COVID-19 control by vaccination or otherwise and subsequent eradication. As mutations (synonymous and asynonymous/non-synonymous) occur during every replication cycle, there is every chance that antigenic mutants develop that can escape neutralization (neutralization escape mutants) by neutralizing antibodies elicited by a vaccine candidate. The information generated and available as of the date on non-synonymous mutations in the structural protein regions of the SARS-CoV-2 are many; some of these are mentioned in brief subsequently. Therefore, amino acid replacements observed in the Spike gene and elsewhere can alter the antigenic nature of the (SARS-CoV-2) virus as a whole; and while identifying/ selecting a virus isolate/ population for production of COVID-19 vaccine, two-way antigenic relationships between a set of selected virus isolates need to be done (employing serum antibodies/ polyclonal antibodies) in order to identify the most (antigenically) dominant virus isolate for development of an efficacious vaccine, as being done in case of FMD vaccine. To summarise, the experiences gained in the control and eradication of FMD in livestock and the technologies adapted therein with success, viz., (1) VIA antibody assay, (2) vaccine strain selection by two-way antigenic profiling, and (3) vaccine matching exercise from time to time need to be adapted to control COVID-19 in the shortest possible time. Further, as in FMD, the application of Camelid (Dromedary) nanobodies (nano antibodies) [12] holds promise in developing highly specific and sensitive serological assays for COVID-19 with negligible cross-reactions. It is to add that, once two-way antigenic match and vaccine matching exercise is adapted, it is likely that (1) different virus strains would be required in different epidemiological areas/ ecology or (2) there could be the necessity of having multivalent (more than one virus antigen) preparations to control the COVID-19 and subsequent eradication of the virus. The details of FMD vaccine design, country/region-wise, and application of companion diagnostics may be obtained from the OIE (World Organisation 
for Animal Health), Paris. Application of FMD control strategies to COVID-19 will be a new dimension of "One Health".

\subsection{Phylogenetic analysis and antigenicity of SARS-CoV-2.}

Phylogenetic analysis of SARS-CoV-2 strains revealed 3 major clades, including S, V, and G, and 2 subclades (G.1 and G.2). There were 767 types of synonymous and 1,352 types of non-synonymous mutation [13]. Mutations in ORF1a, ORF1b, S, and N genes were detected at high frequency, whereas ORF7b and E genes exhibited low frequency. The names $\mathrm{S}, \mathrm{G}$, and $\mathrm{V}$ clades are assigned by GISAID, according to amino acid substitutions found in Orf $8, \mathrm{~S}$, and 3a [14]. Direct genome sequencing of the SARS-CoV-2 genome from nasopharyngeal and oropharyngeal swab samples of infected individuals in Eastern India revealed the presence of A2a and B4 clades [15]. In Taiwan, genomes of 5 strains sequenced from clustered infections were classified into a new clade with ORF1ab-V378I mutation and 3 dominant clades ORF8L84S ORF3a-G251V and S-D614G [16]. Taiwanese viruses were classified into various clades, including a new ORF8-deletion mutant. Genome sequence analysis revealed that the cat SARS-CoV-2 belongs to the phylogenetic clade A2a-like most French isolates of human SARS-CoV-2 [17]. Analysis of complete genome sequences of 64 SARS-CoV-2 strains isolated from 30 December 2019 to 9 March 2020 in Europe, North and South America, and South East Asia revealed substitutions in different genome regions viz., 5'non coding region (5'NCR), 1a, 1b, S, 3a, M, 8 and $\mathrm{N}$. The Clade 1 strain shared the same substitution $\mathrm{C} \rightarrow \mathrm{T}$ in $5^{\prime}$ NCR, 1a, 1b, and $\mathrm{S}$ genes, the clade 2 strains shared the same substitutions in 1a and 8 genes, and clade 3 strains shared the same substitutions in 1a and 3a genes [14]. Analysis of 4,894 SARS-CoV-2 complete genome sequences [18] identified 11 residues with high-frequency substitutions, including four of these having potential for positive selection. These fastevolving sites are in the non-structural proteins nsp2, nsp5 (3CL-protease), nsp6, nsp12 (polymerase), and nsp13 (helicase), in accessory proteins (ORF3a, ORF8) and the structural proteins $\mathrm{N}$ and $\mathrm{S}$. Structural analysis revealed that these mutations are located on the surface of the proteins that modulate biochemical properties.

\subsection{Spike protein.}

The spike protein (S; ORF2) mediates receptor binding and membrane fusion and is crucial for host tropism and virus transmission, and also acquired immune protection. It has been reported that the binding of the SARS-CoV RBD to the ACE2 receptor were variable in the SARS-CoV-2 RBD domain, including Asn439 and 501, Gln 493, Gly 485 and Phe 486 [9]. The Spike (S) gene of SARS-CoV-2 has two domains, S1 and S2. The S1 domain is involved in host cell receptor recognition and binding, whereas the S2 domain is involved in triggering viral fusion with the host cell membrane. Spike protein (D614G) has evolved and quickly eclipsed the original Wuhan strain in terms of prevalence [19]. This Spike variation is found in the most common clade (A2a) of SARS-CoV-2 genomes worldwide. Several researchers have argued that the delta and delta plus mutant variants confer greater transmissibility, resulting in a positive selection based on phylogenomic data. The delta and delta plus mutant mutations in the Spike protein boost human cell transduction [19]. There is a preponderance of G614 variants. Distinct viral clades have a likely impact on COVID-19 pathogenesis and spread. Sequence analysis from 2310 viral isolates revealed that residue at 614 of the viral spike protein is changed from a putative ancestral aspartic acid (D) to glycine $(G)$ between two viral clades 
in the USA [20]. The presence of A2a clade that is characterized by P323L in RNA-dependent RNA polymerase and delta and delta plus mutant in the $S_{D}$ domain of the Spike (S) glycoprotein has also been detected in India [15]. There were also novel subclones having a nonsynonymous mutation of G1124V in the S2 gene [15]. In the receptor-binding domain (RBD) of the $\mathrm{S}$ gene, 11 non-synonymous mutations were observed in the region adjacent to the angiotensin-converting enzyme 2 (ACE2) binding site [13]. The Mab HA001 identified A475 and F486 in the SARS-CoV-2 RBD, representing new binding sites for neutralizing antibodies [21]. The latest significant $S$ variants identified in the second wave in India and elsewhere are B.1.617 and its Kappa ( $\kappa)$, Delta $(\delta)$, and Delta plus ( $\delta+$; Lys to Asn at position 417) strains, in the order of appearance.

\subsection{Receptor Binding Domain (RBD) and Receptor Binding Motif (RBM) in Spike protein} $(S 1)$.

The receptor-binding motif (RBM) is the main functional region in RBD and is composed of two regions (region 1 and region 2) that form the interface between the $S$ protein and human ACE2 receptor [21]. The region outside the RBM also plays an important role in maintaining the structural stability of the RBD. They demonstrated that SARS-CoV-2 has two types of amino acid residues to maintain its binding affinity with human ACE2 receptor; binding was enhanced by introducing amino acid changes at P499, Q493, F486, A475, and L455, and receptor binding was diminished by replacing residues N501, Q498, E484, T470, K452, and R439. Animal immunization studies revealed that the RBDs of SARS-CoV and SARS-CoV-2 are potential antigens that induce strong clade-specific neutralizing antibodies in mice with weaker cross-neutralizing activity. Six single-amino acid substitutions in RBD of SARS-CoV-2, viz. N501, Q498, E484, T470, K452, and R439, resulted in the loss of interactions with human ACE2 receptors. Five single amino acid substitutions, viz., P499, Q493, F486, A475, and L455 in SARS-CoV-2 RBD, enhanced hACE2 binding activity. Data indicated six asynonymous mutations in the RBD of SARS-CoV-2, viz., N439/R426, L452/K439, T470/N457, E484/ P470, Q498/Y484, and N501/T487, led to enhanced binding affinity of the virus to human ACE2 receptor. These natural mutations in the S protein help monitor the increased infectibility of the virus during transmission [21].

\subsection{Nucleoprotein gene $(N)$.}

Novel subclones were having R203K, and G204R in the SR-rich region of the nucleoprotein $(\mathrm{N})$ gene involved in the viral capsid formation have been reported in Indian isolates [15]. The SR-rich (serine-arginine-rich) region in the $\mathrm{N}$ gene is involved in viral capsid formation.

\subsection{Open Reading Frame 8 (ORF 8).}

A 382-nucleotides deletion in ORF8 (deletion mutant) was found in a Taiwanese strain isolated from a patient who had a travel history to Wuhan [16].

\subsection{Polyprotein lab (pp lab).}

The polyprotein $1 \mathrm{ab}(\mathrm{pp} 1 \mathrm{ab})$ gene is located at the 5' end of the SARS-CoV-2 genome. It is a virulence factor that inhibits host gene expression [22]. Comparison of pp1ab proteins 
of 144 isolates of SARS-CoV-2 from patients around the world revealed that the viral pp1ab has not changed in most isolates throughout the outbreak time; however, a deletion of 8 amino acids (32 to 39 aa; 32GDSVEEVL 39; GISAID database) in the virulence factor nsp 1 was found in a virus isolated from a Japanese patient without critical symptoms. Compared to other beta-coronaviruses, a 42 amino acid signature was found present only in SARS-CoV-2. The signature "DSQQTVGQQDGSEDNQTTTIQTIVEVQPQLEMELTPVVQTIE" is placed between the amino acids 983 and 1024 of pp1ab that corresponds to the N-terminal of papainlike protease.

\section{7. $R N A$-dependent $R N A$ polymerase ( $R d R p)$.}

Seven mutations in RdRp (RNA-dependent RNA polymerase enzyme) of Indian isolates of SARS-CoV-2 have been reported [23]. The secondary structure prediction analysis of these seven mutations showed that three of them cause alteration in the structure of RdRp and can potentially alter the stability of the RdRp protein. They have proposed that $R d R p$ mutations in Indian SARS-CoV-2 isolates might have functional consequences that can interfere with RdRp targeting pharmacological agents.

\section{Foot-and-Mouth Disease and the FMD Virus}

Foot-and-mouth disease (FMD) is an infectious disease caused by the FMD virus that affects cloven-hoofed animals, including domestic and wild bovids, pigs, and elephants. The incubation period of FMD ranges between one and 12 days [24]. The virus can be transmitted by close contact, long-distance aerosol spread, and fomites may aid in its spread. The same is applicable to COVID-19.

The FMD virus (FMDV) assigned to the genus Apthovirus in the family Picornaviridae is a non-enveloped, positive-sense, single-stranded RNA virus of approximately $8.5 \mathrm{~kb}$ in length. The virus has an icosahedral symmetry with a capsid containing 60 copies of each of the structural proteins [VP4, VP2, VP3, and VP1] (Figure 1) [25, 26]. The VP1-3 protein is surface exposed, and VP4 is internal. Immunodominant epitopes are located on the surfaceexposed interconnecting loops. The Aphthovirus genus is comprised of viruses that primarily infect the upper respiratory tract. There are five antigenic sites, of which site $1(\mathrm{G}-\mathrm{H}$ loop) is linear and trypsin-sensitive, and the rest are conformational and trypsin-resistant [27]. There are seven serotypes of FMDV, viz., A, O, C, Asia 1, SAT 1-3, that is genetically and antigenically distinct with no cross-protection between the serotypes. The viral genome comprises 7250-8203 nt (5'-UTR: up to $1112 \mathrm{nt}$; ORF: 6657-7020 nt; 3'-UTR: 32-110 nt). There is a poly $(\mathrm{C})$ tract close to the $5^{\prime}$-terminus of the genome and varies from 100 to more than $400 \mathrm{nt}$. There is a series of 3-4 pseudoknots in the poly $(\mathrm{C})$ tract comprising of perfectly repeated sequences, each consisting of 21 bases. The genome organisation is: VPg-5'-UTR ${ }^{I R E S}$ II-[L pro/1A-1B-1C-1D-2A npgp/2B-2C/3A-3B1-(3B2-3B3)-3C-3D]-3'-UTR-poly(A).

The deduced polyproteins range from 2,218-2,339 aa. Translation starts at two alternative in-frame initiation sites, resulting in two forms of the $\mathrm{L}$ protein (Lab and $\mathrm{Lb}$ ). $\mathrm{L}$ is a papain-like cysteine proteinase that cleaves itself from the virus polyprotein. The $2 \mathrm{~A}$ polypeptide is very short (chain length $=18$ aa in FMDV), and is involved in NPGP-dependent polypeptide chain interruption at its C-terminus. The genome of FMDV encodes 3 species of VPg. FMD virus is highly antigenically variable, and several studies have reported such variations in all serotypes of FMDV. Serotype $O$ virus is present in all parts of the world 
wherever FMD is endemic [27]. Many vaccination programs are critical because of the antigenic diversity/variation of candidate viruses used in the vaccine formulations [28]. It is reported that antigenicity of serotype $\mathrm{O}$ virus during the serial passage changes significantly.

The isolate showed a poor antigenic match (relationship value $<0.3$ ) initially with the serotype $O$ vaccine strain, which got increased in relationship value after serial passages. Sequencing of capsid region of serotype $O$ virus revealed substitution at four positions $\left(\mathrm{VP} 3: \mathrm{K}_{58} \rightarrow \mathrm{E}\right.$ and $\mathrm{P}_{158} \rightarrow \mathrm{S}, \mathrm{VP} 1: \mathrm{E}_{83} \rightarrow \mathrm{K}$ and $\mathrm{R}_{172} \rightarrow \mathrm{Q}$ ) acquired during the serial passage. Amino acid substitution at position VP3-58 that was identified earlier as crucial for antigenic site IV is critical in the observed antigenic variability [29]. Similar possibilities do exist for the COVID-19 virus. The evolution of the capsid coding region (P1 region) of FMD serotype A over some time has shown the dominance of VP3 ${ }^{59}$ deletion group. A study showed 38 established residues, 10 positions (VP2 80 and 132; VP3 136 and 195; VP1 145, 147,158, and $199)$ were conserved in all the isolates analyzed. But, the three critical positions $(145,147$, and 158 ) in the highly variable VP1 $\beta \mathrm{G}-\beta \mathrm{H}$ loop were found conserved in the data set used over three decades. Therefore, it can be argued that these 10 positions have not contributed significantly to the natural antigenic variation in the serotype A genotypes. Phylogenetic relationships suggest that genetically similar viruses (with less than $2 \%$ nucleotide difference) belonging to clade $18 \mathrm{a}$ of the VP3 ${ }^{59}$-deletion group have circulated in certain parts of India [30]. With 5\% nucleotide divergence cut off of the isolates of serotype Asia 1 collected from Asian countries during 2003-2007 were classified into six Groups (I-VI) [31]. Pakistan isolates collected during 2008-2009 were classified as Group VII [32]. Indian and Bhutan isolate collected during 2001-2004 and 2002 respectively formed Group III. Isolates collected after 2005 from India, barring just three isolates, were grouped separately (within lineage C), and this novel group has been designated here as Group VIII. The global consensus length of VP1 protein in serotype Asial is 211 amino acids. One codon deletion at the $44^{\text {th }}$ position was observed in lineage $\mathrm{B}$. The deletion is located in the $\mathrm{bB}$ strand of $\mathrm{bB}-\mathrm{bC}$ loop, which is considered to be antigenically critical in other FMDV serotypes. The prototypic strain PAK1/54 also contains this deletion. In two strains, IND321/2001 and IND98/2003 (collected in 2002) of lineage $\mathrm{C}$, one codon insertion at $140^{\text {th }}$ positions in $\mathrm{bG}-\mathrm{bH}$ loop was detected [33].

The process of cell attachment of FMDV is mediated by RGD triplet along with residues at +1 and +4 , and to some extent at +2 downstream to RGD within bG-bH loop of VP1. Two different motifs were found in the majority of the Indian isolates, and a genotypespecific conservation pattern was evident. The motif was found to be RGDXAL in genotype I and RGDMXAL in genotype II. The residue at +3 position also demonstrated a genotypespecific variation to a larger extent ( $\mathrm{V}$ in genotype I and A in genotype II) [32].

\subsection{Selection of candidate vaccine strains.}

The candidate vaccine strain selected from the field isolates/strains should have a broad range of protection against most of the field strains under circulation. The immunity induced by a vaccine prepared using a selected candidate strain depends on its potency, antigenic match with field strains, and vaccine schedule [34, 35]. Sometimes vaccine formulation is warranted to incorporate one or more strains that induce a broad range of protective immunity against multiple factors. 


\subsection{Principles of vaccine strain selection.}

Vaccines prepared from one virus that is antigenically related to field strains should provide strong immunity and match antigenically with circulating virus strains.

\subsection{Vaccine matching tests.}

Determination of the antigenic type of all field strains using specific serological reagents. Monoclonal antibodies and genetic typing can also be used. If there are many field strains, using statistical methods, representative samples can be selected and then compared. By comparing the nucleotide sequences of structural genes of vaccine strains and field isolates, vaccine matches can be predicted, provided amino acid differences are carefully matched. Once the candidate vaccine virus is characterized, in vitro serological methods can be used to compare it with serum obtained from vaccinated animals [35].

Calculation of relationship values (' $r$ ' values) between FMD viruses using pools of antisera prepared in bovine against each vaccine strain to be matched $S$ is the best method; the antigenic similarity between vaccines and field isolates is estimated from their comparative reactivity with the appropriate serum pool. If VNT is used [36], the neutralizing titers of the BVS against 100 TCID50 (tissue culture infecting dose) of the homologous vaccine strain and the same dose of a field isolate are compared determine how antigenically 'similar' the field virus is to the vaccine strain. In the neutralization test, one-way comparative values (' $r_{1}$ ' values) greater than 0.3 indicate that the field isolate is sufficiently similar to the vaccine strain for the vaccine to be likely to confer protection against challenge with the field isolate [37]. Similar methodology may also be applicable to COVID-19.

\section{Conclusions}

Both FMD virus and SARS-CoV-2 are transboundary agents with similarities in genome type and replication process. FMD is an infectious and sometimes fatal viral disease that affects cloven-hoofed animals, including domestic and wild bovids. In addition to severe clinical manifestations, both the diseases can be asymptomatic. Though carrier status and persistent infection occur in FMD vaccinated and convalescent animals, with no clinical symptoms that pose a threat to FMD control, a similar situation may arise once COVID-19 vaccination is implemented in all countries. There is little information available on the possibility of carrier status and persistent infection in COVID-19 convalescent individuals. There is a necessity to have specific and sensitive anti-NSP antibody assay(s) to monitor virus persistence/ carrier status in such individuals. Antibody response to NSPs will help in differentiating healthy individuals from infected ones. Comparing the kinetics of antibody response to SPs and NSPs will help differentiate vaccinated individuals from those superinfected following vaccination. It is most likely that the antibodies to NSP(s) will be detectable for longer periods in the blood/ serum than antibodies against structural proteins in persistently infected humans, as in the case of FMD. Further, the continual presence of antiNSP antibodies without clinical sickness will certainly indicate persistent virus infection/ carrier status/ asymptomatic infection, which may occur in both COVID-19 vaccinated and convalescent humans. However, anti-NSP assays will be highly essential in monitoring virus spread and elimination. Application of the principles of Progressive Control Pathway for Footand-Mouth Disease (PCP-FMD) to COVID-19 control will be beneficial. The PCP-FMD developed jointly by the FAO and The European Commission for the Control of Foot-and- 
Mouth Disease (EuFMD) and endorsed by the world organization for animal health (OIE), guides endemic countries through a series of incremental steps (Steps 0 to 5) to better manage FMD risks aiming at control and eradication of the disease. Maybe we lack the quality standards of the laboratory results of COVID-19, as right now, we do not have any "proficiency testing" platform to develop and ascertain comparable proficiency in conducting laboratory analyses at different places and laboratories in the world. The PCP recognizes that there are differences in the risk of infection between different countries. Looking at the global presence of the COVID-19, we may need to adapt strategy similar to PCP-FMD to control COVID-19, that requires following competency/ data and information, viz., (1) information on epidemiology including geographical distribution of the disease within and between countries, (2) availability of suitable laboratory diagnostic tests, (3) antigenic nature of the circulating virus strains, (4) availability of suitable vaccine doses with appropriate vaccine strains antigenically matching $>80 \%$ of the field outbreak strains, (5) basal antibody status of the target population, (6) identification of population to be vaccinated and frequency of re-vaccination, (7) post-vaccination sero-monitoring, (8) companion diagnostics including anti-NSP antibody assays for active and passive surveillance of the disease in vaccinated and unvaccinated populations, as well as to differentiate antibody response elicited by virus infection from vaccination and identify carrier status, and (9)vaccine matching of outbreak strains to ensure antigenic coverage of the vaccine in use. Looking at the current upward rising in the number of COVID-19 positive humans (by RT-PCR and antigen tests) in India (as of 27 July 2020) and being at number 3 in the world, following the USA and Brazil, indicates the possibility of the establishment of persistent infection and endemicity in the near future. Once the SARSCoV-2 becomes endemic, there will be a tough time controlling the disease and transmission of the causative virus by vaccination. Much more time will be needed to clear the virus from the human population in any epidemiological way setting.

\section{Funding}

This research received no external funding.

\section{Acknowledgments}

The information available on the Internet (NCBI-PubMed, WHO, OIE, ICMR, ICAR, Govt of India, Wikipedia, Newspaper and News periodicals etc.) have been incorporated in the present review. We sincerely acknowledge each of these.

\section{Conflicts of Interest}

The authors declare that they do not have any conflict of interest. All authors have equally contributed.

\section{References}

1. Mahy, B. W. J. Introduction and History of Foot-and-Mouth Disease Virus. Springer-Verlag 2005, 288, 1-8, https://link.springer.com/chapter/10.1007\%2F3-540-27109-0_1.

2. Loeffier, F.; Frosch, P. Summarischer Bericht Ober die Ergebnisse der Untersuchungen der Kommission zur Erforschung der Maul-und Klauenseuche bei dem Institut for Infektionskrankheiten in Berlin Zentralblatt Fur Bakteriologie Mikrobiologie Und Hygiene. Series A-Medical Microbiology Infectious Diseases Virology Parasitology 1897 , 1, 257-259, 
3. Acharya, R.; Fry, E.; Stuart, D.; Fox, G.; Rowlands, D.; Brown, F. The 3-dimensional structure of foot-andmouth disease virus at 2.9- $\AA$ resolution. Nature 1989, 337, 709-716, https://doi.org/10.1038/337709a0.

4. Vijgen, L. Keyaerts Els, P.Lemey, P. Maes, K.V. Reeth, N. Hans, P. Maurice, M.V. Ranst, Evolutionary History of the Closely Related Group 2 Coronaviruses: Porcine Hemagglutinating Encephalomyelitis Virus, Bovine Coronavirus, and Human Coronavirus OC43. Journal of Virology 2006, 80, 7270-7274, https://doi.org/10.1128/JVI.02675-05.

5. Vijgen, L.; Keyaerts, E.; Elien, M.; Inge, T.; Elke,W.; Philippe, L.; Vandamme-Anne, M.; Marc, V.R. Complete Genomic Sequence of Human Coronavirus OC43: Molecular Clock Analysis Suggests a Relatively Recent Zoonotic Coronavirus Transmission Event. Journal of Virology 2005, 79, 1595-1604, https://doi.org/10.1128/JVI.79.3.1595-1604.2005.

6. Gorbalenya, A.E.; Baker, S.C.; Baric, R.S.; de Groot, R.J.; Drosten, C.; Gulyaeva, A.A.; Haagmans, B.L.; Lauber, C.; Leontovich, A.M.; Neuman, B.W.; Penzar, D.; Perlman, S.; Poon, L.L.M.; Samborskiy, D.V.; Sidorov, I.A.; Sola, I.; Ziebuhr, J. The species Severe acute respiratory syndromerelated coronavirus: classifying 2019-nCoV and naming it SARS-CoV-2 (2020). Nature Microbiology 2020, 5, 536-544, https://doi.org/10.1038/s41564-020-0695-z.

7. Benvenuto, D.; Giovanetti, M.; Salemi, M.; Prosperi, M.; De Flora, C.; Alcantara, L.C.J;. Angeletti, S.; Ciccozzi, M. The global spread of 2019-nCoV: a molecular evolutionary analysis. Patho and Global Health 2020, 114, 64-67, https://doi.org/10.1080/20477724.2020.1725339.

8. Salemi, M.; Fitch, W. M.; Ciccozzi, M.; Alvarez, M. J. R.; Rezza, G.; Lewis, M. J. Severe Acute Respiratory Syndrome Coronavirus Sequence Characteristics and Evolutionary Rate Estimate from Maximum Likelihood Analyis. J Virology 2004, 78, 1602-3, https://doi.org/10.1128/jvi.78.3.1602-1603.2004.

9. Lu, R.; Zhao, X.; Li, J.; Niu, P.; Yang, B.; Wu, H.; Wang, W.; Song, H.; Huang, B.; Zhu, N.; Bi, Y.; Ma, X.; Zhan, F.; Wang, L.; Hu, T.; Zhou, H.; Hu, Z.; Zhou, W.; Zhao, L.; Chen, J.; Meng, Y.; Wang, J.; Lin, Y.; Yuan, J.; Xie, Z.; Ma, J.; Liu, W.J.; Wang, D.; Xu, W.; Holmes, E.C.; Gao, G.F.; Wu, G.; Chen, W.; Shi, W.; Tan, W. Genomic characterisation and epidemiology of 2019 novel coronavirus: implications for virus origins and receptor binding. The Lancet 2020, 395, 565-574, https://doi.org/10.1016/S0140-6736(20)30251-8.

10. Domingo, E.; Escarmı's, C.; Baranowski, E.; Ruiz-Jarabo, C.M.; Carrillo, E.; Nu'ñez, J.I.; Sobrino, F. Evolution of foot-and-mouth disease virus. Virus Res 2003, 91, 47-63, https://doi.org/10.1016/s01681702(02)00259-9.

11. Huang, A.T.; Carreras, B. G.; Hitchings, M. D. T.; Yang, B.; Katzelnick, L. C.; Rattigan, S. M.; Borgert, B. Moreno, A. C. A.; Solomon, B. D.; Barraquer, I.R.; Lessler, J.; Salje, H.; Burke, D.; Wesolowski, A.; Cummings, D.A.T. A systematic review of antibody mediated immunity to coronaviruses: antibody kinetics, correlates of protection, and association of antibody responses with severity of disease. medRxiv preprint 2020, https://doi.org/10.1101/2020.04.14.20065771.

12. Dash, L.; Subramaniam, S.; Khulape, S.A.; Prusty, B. R.; Pargai, K.; Narnaware, S.D.; Patil, N. V.; Pattnaik, B. Development and Utilization of VHH AntibodiesDerived from Camelus Dromedarius Against Foot-andMouth Disease Virus. Animal biotechnology 2019, 30, 57-62, https://doi.org/10.1080/10495398.2018.1433191.

13. Kim, J.S.; Jang, J.H.; Kim, J.M.; Chung, Y.S.; Yoo, C.K.; Han, M.G. Genome-Wide Identification and Characterization of Point Mutations in the SARS-CoV-2 Genome. Osong Public Health Res Perspect 2020, 11, 101-111, https://doi.org/10.24171/j.phrp.2020.11.3.05.

14. Castells, M.; Tort, F.L.; Colina, R.; Cristina, J. Evidence of increasing diversification of emerging SARSCov-2 strains. J Medical Virology 2020, https://doi.org/10.1002/jmv.26018.

15. Maitra, A.; Sarkar, M.C.; Raheja, H.; Biswas, N.K.; Chakraborti, S.; Singh, A.K.; Ghosh, S.; Sarkar, S.; Patra, S.; Mondal, R.K.; Ghosh, T.; Chatterjee, A.; Banu, H.; Majumdar, A.; Chinnaswamy, S.; Srinivasan, N.; Dutta, S.; Das, S. Mutations in SARS-CoV-2 viral RNA identified in Eastern India: Possible implications for the ongoing outbreak in India and impact on viral structure and host susceptibility. J. Biosci. (Bangalore) 2020, 45, 76, https://doi.org/10.1007/s12038-020-00046-1.

16. Gong, Y.-N.; Tsao, K.-C.; Hsiao, M.-J.; Huang, C.-G.; Huang, P.-N.; Huang, P.-W.; Lee, K.-M.; Liu, Y.-C.; Yang, S.-L.; Kuo, R.-L.; Chen, K.-F.; Liu, Y.-C.; Huang, S.-Y.; Huang, H.-I.; Liu, M.-T.; Yang, J.-R.; Chiu, C.-H.; Yang, C.-T.; Chen, G.-W.; Shih, S.-R. SARS-CoV-2 genomic surveillance in Taiwan revealed novel ORF8-deletion mutant and clade possibly associated with infections in Middle East. Emerging Microbes \& Infections 2020, 9, 1457-1466, https://doi.org/10.1080/22221751.2020.1782271.

17. Sailleau, C.; Dumarest, M.; Vanhomwegen, J.; Delaplace, M.; Caro, V.; Kwasiborski, A.; Hourdel, V.; Chevaillier, P.; Barbarino, A.; Comtet, L.; Pourquier, P.; Klonjkowski, B.; Manuguerra, J.-C.; Zientara, S.; 
Le Poder, S. First detection and genome sequencing of SARS-CoV-2 in an infected cat in France. Transbound. Emerg. Dis. 2020, 67, 2324-2328, https://doi.org/10.1111/tbed.13659.

18. Zhao, J.; Sun, J.; He, W.-T.; Ji, X.; Gao, Q.; Zhai, X.; Suchard, M.A.; Hong, S.L.; Baele, G.; Su, S.; Zhou, J.; Veit, M. Snapshot of the evolution and mutation patterns of SARS-CoV-2. BioRxiv 2020, https://doi.org/10.1101/2020.07.04.187435.

19. Daniloski, Z.; Jordan, T.X.; Ilmain, J.K.; Guo, X.; Bhabha, G.; Sanjana, N.E. The Spike D614G mutation increases SARS-CoV-2 infection of multiple human cell types. Elife 2021, 10, e65365, https://elifesciences.org/articles/65365.

20. Brufsky, A. Distinct viral clades of SARS-CoV-2: Implications for modeling of viral spread. J. Med. Virol. 2020, 92, 1386-1390, https://doi.org/10.1002/jmv.25902.

21. Yi, C.; Sun, X.; Ye, J.; Ding, L.; Liu, M.; Yang, Z.; Lu, X.; Zhang, Y.; Ma, L.; Gu, W.; Qu, A.; Xu, J.; Shi, Z.; Ling, Z.; Sun, B. Key residues of the receptor binding motif in the spike protein of SARS-CoV-2 that interact with ACE2 and neutralizing antibodies. Cell Mol Immunol 2020, 17, 621-630, https://doi.org/10.1038/s41423-020-0458-z.

22. Cárdenas-Conejo, Y.; Liñan-Rico, A.; García-Rodríguez, D.A.; Centeno-Leija, S.; Serrano-Posada, H. An exclusive 42 amino acid signature in pplab protein provides insights into the evolutive history of the 2019 novel human-pathogenic coronavirus (SARS-CoV-2). J. Med. Virol. 2020, 92, 688-692, https://doi.org/10.1002/jmv.25758.

23. Chand, G. B.; Banerjee, A.; Azad, G. K. Identification of novel mutations in RNA-dependent RNA polymerases of SARS-CoV-2 and their implications on its protein structure. Peer J 2020, 3, e9492, https://doi.org/10.7717/peerj.9492.

24. Arzt, J.; Pacheco, J.M.; Rodriguez, L. L. The early pathogenesis of foot-and-mouth disease in cattle after aerosol inoculation. Identification of the nasopharynx as the primary siteof infection. Veterinary Pathology 2010, 47, 1048-1063, https://doi.org/10.1177/0300985810372509.

25. Belsham, G.J. Distinctive features of foot-and-mouth disease virus, a member of the picornavirus family; aspects of virus protein synthesis, protein processing and structure. Progress in biophysics and molecular biology 1993, 60, 241-60, https://doi.org/10.1016/0079-6107(93)90016-d.

26. M.J. Grubman, B. Baxt, Foot-and-mouth disease. Clinical microbiology reviews 2004, 17, 465-93, https://doi.org/10.1128/CMR.17.2.465-493.2004.

27. Pacheco, J.M.; Smoliga, G.R.; O’Donnell, V.; Brito, B.P.; Stenfeldt, C.; Rodriguez, L.L.; Arzt, J. Persistent Foot-and-Mouth Disease Virus Infection in the Nasopharynx of Cattle; Tissue-Specific Distribution and Local Cytokine Expression. PLoS One 2015, 10, e0125698, https://doi.org/10.1371/journal.pone.0125698.

28. Gebauer, F.; De La Torre, J.C.; Gomes, I.; Mateu, M.G.; Barahona, H.; Tiraboschi, B.; Bergmann, I.; De Mello, P.A.; Domingo, E. Rapid selection of genetic and antigenic variants of foot-and-mouth disease virus during persistence in cattle. J. Virol. 1988, 62, 2041-2049, https://doi.org/10.1128/JVI.62.6.2041-2049.1988.

29. Subramaniam, S.; Das, B.; Biswal, J.K.; Ranjan, R.; Pattnaik, B. Antigenic variability of foot-and-mouth disease virus serotype $\mathrm{O}$ during serial cytolytic passage. Virus Genes 2017, 53, 931-934, https://doi.org/10.1007/s11262-017-1494-3.

30. Mohapatra, J.K.; Subramaniam, S.; Pandey, L.K.; Pawar, S.S.; De, A.; Das, B.; Sanyal, A.; Pattnaik, B. Phylogenetic structure of serotype A foot-and-mouth disease virus: global diversity and the Indian perspective. 2011, 92, 873-879, https://doi.org/10.1099/vir.0.028555-0.

31. Valarcher, J.-F.; Knowles, N.J.; Zakharov, V.; Scherbakov, A.; Zhang, Z.; Shang, Y.-J.; Liu, Z.-X.; Liu, X.T.; Sanyal, A.; Hemadri, D. Multiple origins of foot-and-mouth disease virus serotype Asia 1 outbreaks, 2003-2007. Emerging Infect. Dis. 2009, 15, 1046, https://doi.org/10.3201/eid1507.081621.

32. Jamal, S.M.; Ferrari, G.; Ahmed, S.; Normann, P.; Belsham, G.J. Molecular characterization of serotype Asia1 foot-and-mouth disease viruses in Pakistan and Afghanistan; emergence of a new genetic Group and evidence for a novel recombinant virus. Infect., Genet. Evol. 2011, 11, 2049-2062, https://doi.org/10.1016/j.meegid.2011.09.015.

33. Subramaniam, S.; Mohapatra, J.K.; Sharma, G.K.; Das, B.; Dash, B.B.; Sanyal, A.; Pattnaik, B. Phylogeny and genetic diversity of foot and mouth disease virus serotype Asia1 in India during 1964-2012. Vet. Microbiol. 2013, 167, 280-288, https://doi.org/10.1016/j.vetmic.2013.08.023.

34. OIE (2014) World Organisation for Animal Health (OIE)- Manual of Diagnostic Tests and Vaccines for Terrestrial Animals, 2014. Chapter 2.1.5. Foot-and-mouth disease. OIE, Paris (2014). https://www.oie.int/en/what-we-do/standards/codes-and-manuals/terrestrial-manual-online-access/. 
35. Paton, D.J.; Valarcher, J.F.; Bergmann, I.; Matlho, O.G.; Zakharov, V.M.; Palma, E.L.; Thomson, G.R. Selection of foot and mouth disease vaccine strains - a review. Rev. Sci. Tech. Off. Int. Epiz 2005, 24, 98193, https://pubmed.ncbi.nlm.nih.gov/16642769.

36. Rweyemamu, M.M.; Booth, J.C.; Head, M.; Pay, T.W.F. Microneutralization tests for serological typing and subtyping of foot-and-mouth disease virus strains. J. Hyg. (Lond.) 1978, 81, 107-123, https://doi.org/10.1017/s002217240005381x.

37. Rweyemamu, M.M. Antigenic variation in foot-and-mouth disease: studies based on the virus neutralization reaction. J. Biol. Stand. 1984, 12, 323-337, https://doi.org/10.1016/s0092-1157(84)80013-x. 